Research Article

\title{
On the convergence of triple Elzaki transform
}

\author{
Tarig M. Elzaki ${ }^{1}$ (D) Adil Mousa ${ }^{2}$
}

C The Author(s) 2019 OPEN

\begin{abstract}
In this work, the convergence properties of triple Elzaki transform was examine and the results presented in the form of theorems on convergence, absolute convergence and uniform convergence of triple Elzaki transform. The triple Elzaki transform of triple integral examined for integral evaluation. Finally, Volterra integro-partial differential equation solved by using triple Elzaki transform.
\end{abstract}

Keywords Triple Elzaki transform · Convergence $\cdot$ Absolute convergence $\cdot$ Uniform convergence $\cdot$ Integro-partial differential equations

\section{Introduction}

We have introduced the modified version of Sumudu and Laplace transforms [1, 2], namely Elzaki transform to solve some problems. In the recent years, this transform has become more accurate and efficient. Elzaki transformation [3-11], introduced by Tarig Elzaki in 2011. Elzaki transform solving differential equations with variable coefficients, which are not solved, by Sumudu transform [12-16].

In this paper, we have discussed the various convergence properties of triple Elzaki transformation. The triple Elzaki transform is a very useful technique to solve some differential equations [17-20], partial differential equations, and integral equations. This transform used as a very efficient tool in simplifying the calculations in many disciplines of engineering and mathematics.

Triple Elzaki transform:

Let $f(x, y, t)$ be a function that can be expressed as convergent infinite series, and let $(x, y, t) \in R_{3}^{+}$, then, the triple Elzaki transform is denoted by:

$\mathrm{E}_{3}[\mathrm{f}(\mathrm{x}, \mathrm{y}, \mathrm{t}):(\rho, \mathrm{s}, \delta)]=\rho s \delta \int_{0}^{\infty} \int_{0}^{\infty} \int_{0}^{\infty} \mathrm{e}^{-\frac{x}{\rho}-\frac{y}{\mathrm{~s}}-\frac{\mathrm{t}}{\delta}} f(x, y, t) d x d y d t$, where $x, y, t>0$ and $\rho, s, \delta$ are transform variables for $x, y$ and $t$ respectively, whenever the improper integral is convergent.

\section{Convergence theorems of triple Elzaki integral}

In this section, we prove the convergence theorem of triple Elzaki integral.

Theorem 2.1 If $\emptyset(x, y, t)$ is a continuous function on the positive of the $x, y, t$-plane. If the integral converges at $\rho=\rho_{o^{\prime}}$ $\mathrm{s}=\mathrm{s}_{\mathrm{o}}, \delta=\delta_{\mathrm{o}}$ then, the integral:

$\rho s \delta \int_{0}^{\infty} \int_{0}^{\infty} \int_{0}^{\infty} \mathrm{e}^{-\frac{x}{\rho}-\frac{y}{s}-\frac{t}{\delta}} \emptyset(x, y, t) d x d y d t$

Converges for at $\rho<\rho_{\circ}, s<s_{\circ}, \delta<\delta_{\circ}$ For the proof, we will use the following lemmas.

Lemma 2.2 If the integral,

$\checkmark$ Tarig M. Elzaki, tarig.alzaki@gmail.com, tfarah@uj.edu.sa; Adil Mousa, aljarada@gmail.com | ${ }^{1}$ Mathematics Department, Faculty of Sciences and Arts-Alkamil, University of Jeddah, Jeddah, Saudi Arabia. ${ }^{2}$ Department of Mathematics, Faculty of Science and Technology, Omdurman Islamic University, Khartoum, Sudan. 
$s \int_{0}^{\infty} \mathrm{e}^{-\frac{y}{s}} \emptyset(x, y, t) d y$

converges at $\mathrm{s}=\mathrm{s}_{\mathrm{o}}$ then the integral converges for $\mathrm{s}<\mathrm{s}_{\circ}$.

$s \int_{0}^{\infty} \mathrm{e}^{-\frac{y}{s}} \emptyset(x, y, t) d y=\left(\frac{\mathrm{s}_{\circ}-\mathrm{s}}{\mathrm{s}_{\circ}^{2}}\right) \int_{0}^{\infty} \mathrm{e}^{-\left(\frac{\mathrm{s}_{0}-\mathrm{s}}{s s_{0}}\right) y} \alpha(x, y, t) d y$

Proof Consider the set,

$\alpha(x, y, t)=\mathrm{s}_{\circ} \int_{0}^{y} \mathrm{e}^{-\frac{u}{s_{\circ}}} \emptyset(x, u, t) \mathrm{du}, \quad(0<y<\infty)$

Therefore $\alpha(\mathrm{x}, 0, \mathrm{t})=0$ and $\lim _{y \rightarrow \infty} \alpha(x, y, t)$ exist because, the integral $s \int_{0}^{\infty} \mathrm{e}^{-\frac{y}{s}} \emptyset(x, y, t) d y$ converges at $s=s_{\circ}$, by a fundamental theorem of calculus $[17,18]$ we have:

$\alpha_{y}(x, y, t)=\mathrm{s}_{0} \emptyset(x, v, t) \mathrm{e}^{-\frac{y}{s_{0}}}$ hence the integral,

choose $\epsilon_{1}$ and $\mathrm{R}_{1}$ such that $\left(0<\epsilon_{1}<\mathrm{R}_{1}\right)$, then:

$\begin{aligned} s \int_{\epsilon_{1}}^{\mathrm{R}_{1}} \mathrm{e}^{-\frac{y}{s}} \emptyset(x, y, t) d y & =\frac{\mathrm{s}}{\mathrm{s}_{\circ}} \int_{\epsilon_{1}}^{\mathrm{R}_{1}} \mathrm{e}^{-\frac{y}{s}} \alpha_{y}(x, y, t) \mathrm{e}^{\frac{y}{s_{0}}} d y \\ & =\frac{\mathrm{s}}{\mathrm{s}_{\circ}} \int_{\epsilon_{1}}^{\mathrm{R}_{1}} \mathrm{e}^{-\left(\frac{\mathrm{s}_{0}-s}{s s_{\circ}}\right) y} \alpha_{y}(x, y, t) d y,\end{aligned}$

$s \int_{0}^{\infty} \mathrm{e}^{-\frac{y}{s}} \emptyset(x, y, t) d y$, converges for, $\mathrm{s}<\mathrm{s}_{\circ}$.

Lemma 2.3 If the integral,

by using integration by parts

the theorem proved if the integral on the right converges.

Directly by using the limit test for convergence (see [17]) we see,

$$
\begin{aligned}
\lim _{y \rightarrow \infty} y^{2} \mathrm{e}^{-\left(\frac{s_{0}-s}{s s_{0}}\right) y} \alpha(x, y, t) & =\lim _{y \rightarrow \infty} \frac{y^{2}}{\mathrm{e}^{\left(\frac{s_{0}-s}{s s_{0}}\right) y}}\left[\lim _{y \rightarrow \infty} \alpha(x, y, t)\right] \\
& =0 *\left[\lim _{y \rightarrow \infty} \alpha(x, y, t)\right]=0,
\end{aligned}
$$

$h(x, s, t)=s \int_{0}^{\infty} e^{-\frac{y}{s}} \emptyset(x, y, t) d y$,

converges for $\mathrm{s} \leq \mathrm{s}$, and integral,

$$
\begin{aligned}
& =\frac{\mathrm{s}}{\mathrm{s}_{\circ}}\left\{\left|\mathrm{e}^{-\left(\frac{s_{0}-s}{s s_{0}}\right) y} \alpha(x, y, t)\right|_{\epsilon_{1}}^{\mathrm{R}_{1}}-\int_{\epsilon_{1}}^{\mathrm{R}_{1}} \mathrm{e}^{-\left(\frac{s_{0}-s}{s s_{0}}\right) y}\left[-\left(\frac{\mathrm{s}_{\circ}-\mathrm{s}}{\mathrm{ss}}\right)\right] \alpha(x, y, t) d y\right\} \\
& s \int_{\epsilon_{1}}^{\mathrm{R}_{1}} \mathrm{e}^{-\frac{y}{s}} \emptyset(x, y, t) d y=\frac{\mathrm{s}}{\mathrm{s}_{\circ}}\left\{\mathrm{e}^{-\left(\frac{\mathrm{s}_{0}-s}{s s_{0}}\right) R_{1}} \alpha\left(x, \mathrm{R}_{1}, \mathrm{t}\right)-\mathrm{e}^{-\left(\frac{s_{0}-s}{s s_{0}}\right) \epsilon_{1}} \alpha\left(x, \epsilon_{1}, \mathrm{t}\right)+\left(\frac{\mathrm{s}_{\circ}-\mathrm{s}}{\mathrm{s} s_{\circ}}\right) \int_{\epsilon_{1}}^{\mathrm{R}_{1}} \mathrm{e}^{-\left(\frac{s_{0}-s}{s s_{0}}\right) y} \alpha(x, y, t) d y\right\},
\end{aligned}
$$

now let $\epsilon_{1} \rightarrow 0$, both terms on the right which depend on $\epsilon_{1}$ approach a limit and,

$$
\rho \int_{0}^{\infty} e^{-\frac{x}{\rho}} h(x, s, t) d x
$$

$$
\int_{0}^{\mathrm{R}_{1}} \emptyset(x, y, t) \mathrm{e}^{-\frac{y}{s}} \mathrm{dy}=\frac{\mathrm{s}}{\mathrm{s}_{\circ}}\left\{\mathrm{e}^{-\left(\frac{\mathrm{s}_{0}-\mathrm{s}}{s s_{\circ}}\right) \mathrm{R}_{1}} \alpha\left(x, \mathrm{R}_{1}, \mathrm{t}\right)+\left(\frac{\mathrm{s}_{\circ}-\mathrm{s}}{\mathrm{s} \mathrm{s}_{\circ}}\right) \int_{0}^{\mathrm{R}_{1}} \mathrm{e}^{-\left(\frac{\mathrm{s}_{0}-\mathrm{s}}{s s_{\circ}}\right) \mathrm{y}} \alpha(x, y, t) d y\right\}
$$

and then: let, $R_{1} \rightarrow \infty$. If, $s<s_{0}$, the first term on the right converges at $\rho=\rho_{\circ}$ then the integral (6) converges for $\rho<\rho_{\circ}$ approaches zero, 


\section{Proof}

Let: $\beta(x, s, t)=\rho_{\circ} \int_{0}^{x} e^{-\frac{u}{\rho_{\circ}}} \mathrm{h}(u, s, t) d u \quad 0<x<\infty$,

therefore, $\beta(0, s, t)=0$ and $\lim _{x \rightarrow \infty} \beta(x, s, t)$ exist, because $\rho \int_{0}^{\infty} e^{-\frac{x}{\rho}} \mathrm{h}(\mathrm{x}, \mathrm{s}, \mathrm{t}) \mathrm{dx}$ converges at $\rho=\rho_{\mathrm{o}}$, by using fundamental theorem of calculus Eq. (7), becomes,

$\beta_{x}(x, s, t)=\rho h(x, s, t) e^{-\frac{x}{\rho_{0}}}$,

choose $\epsilon_{2}$ and $R_{2}$ and so that $0<\epsilon_{2}<R_{2}$

$\begin{aligned} \rho \int_{\epsilon_{2}}^{R_{2}} \mathrm{e}^{-\frac{x}{\rho}} \mathrm{h}(\mathrm{x}, \mathrm{s}, \mathrm{t}) \mathrm{dx} & =\frac{\rho}{\rho_{\mathrm{o}}} \int_{\epsilon_{2}}^{\mathrm{R}_{2}} \mathrm{e}^{-\left(\frac{\rho_{0}-\rho}{\rho \rho_{0}}\right) \mathrm{x}} \beta_{x}(x, s, t) d x \\ & =\frac{\rho}{\rho_{\circ}}\left\{\mathrm{e}^{-\left(\frac{\rho_{0}-\rho}{\rho \rho_{0}}\right) R_{2}} \beta\left(\mathrm{R}_{2}, s, t\right)-\mathrm{e}^{-\left(\frac{\rho_{0}-\rho}{\rho \rho_{0}}\right) \epsilon_{2}} \beta\left(\epsilon_{2}, s, t\right)+\left(\frac{\rho_{\circ}-\rho}{\rho \rho_{\circ}}\right) \int_{\epsilon_{2}}^{R_{2}} \mathrm{e}^{-\left(\frac{\rho_{0}-\rho}{\rho \rho_{0}}\right) x} \beta(x, s, t) d x\right\},\end{aligned}$

now let $\epsilon_{2} \rightarrow 0$. Both terms on the right which depend on $\epsilon_{2}$ approach a limit and,

converges at $\delta=\delta_{0}$ then the integral (11) converges for $\delta<\delta_{\circ}$. converges for $\rho \leq \rho_{\circ}$ and the integral

$\delta \int_{0}^{\infty} e^{-\frac{t}{\delta}} k(\rho, y, t) d t$

$\rho \int_{0}^{R_{2}} \mathrm{e}^{-\frac{x}{\rho}} \mathrm{h}(\mathrm{x}, \mathrm{s}, \mathrm{t}) \mathrm{dx}=\frac{\rho}{\rho_{\circ}}\left\{\mathrm{e}^{-\left(\frac{\rho_{\mathrm{o}}-\rho}{\rho \rho_{0}}\right) R_{2}} \beta\left(R_{2}, s, t\right)+\left(\frac{\rho_{\circ}-\rho}{\rho \rho_{\circ}}\right) \int_{0}^{R_{2}} \mathrm{e}^{-\left(\frac{\rho_{0}-\rho}{\rho \rho_{\circ}}\right) x} \beta(x, s, t) d x\right\}$

now let $R_{2} \rightarrow \infty$, If $s<s_{0}$, the first term on the right approaches zero.

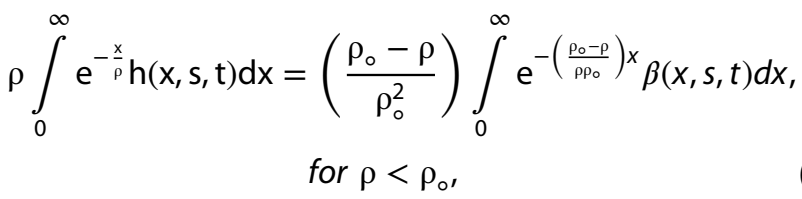

converges.

Directly by means of the limit test for convergence (see [17]) we see,

$$
\begin{aligned}
\lim _{x \rightarrow \infty} x^{2} \mathrm{e}^{-\left(\frac{s_{0}-s}{s s_{0}}\right) x} \beta(x, y, t) & =\lim _{x \rightarrow \infty} \frac{x^{2}}{e^{\left(\frac{s_{0}-s}{s s_{o}}\right) x}}\left[\lim _{x \rightarrow \infty} \beta(x, y, t)\right] \\
& =0 *\left[\lim _{x \rightarrow \infty} \beta(x, y, t)\right]=0,
\end{aligned}
$$

Proof
Let: $\quad \gamma(\rho, y, t)=\delta_{\circ} \int_{0}^{t} e^{-\frac{w}{\delta_{o}}} k(\rho, y, w) d w, \quad 0<t<\infty$,

therefore, $\gamma(\rho, y, 0)=0$ and $\lim _{t \rightarrow \infty} \gamma(x, y, t)$ exist, because $\delta \int_{0}^{\infty} k(\rho, y, t) e^{-\frac{t}{\delta}} d t$ converges at $\delta=\delta_{o}$, by using the fundamental theorem of calculus, Eq. (12), develops as. $\gamma_{t x}(\rho, y, t)=k(\rho, y, w) e^{-\frac{w}{\delta_{0}}}$, choose $\epsilon_{3}$ and $R_{3}$ and so that $0<\epsilon_{3}<R_{3}$ 


$$
\begin{aligned}
\delta \int_{\epsilon 3}^{\mathrm{R} 3} \mathrm{k}(\rho, y, \mathrm{t}) \mathrm{e}^{-\frac{\mathrm{t}}{\delta}} \mathrm{dt} & =\frac{\delta}{\delta_{\circ}} \int_{\epsilon_{3}}^{\mathrm{R}_{3}} e^{-\left(\frac{\delta_{0}-\delta}{\delta \delta_{\circ}}\right) t} \gamma_{t}(\rho, y, \mathrm{t}) d t \\
& =\frac{\delta}{\delta_{\circ}}\left\{\delta^{-\left(\frac{\delta_{0}-\delta}{\delta \delta_{0}}\right) \mathrm{R}_{3}} \gamma\left(\rho, y, \mathrm{R}_{3}\right)-\mathrm{e}^{-\left(\frac{\delta_{0}-\delta}{\delta \delta_{\circ}}\right) \epsilon_{3}} \gamma\left(\rho, y, \epsilon_{3}\right)+\left(\frac{\delta_{\circ}-\delta}{\delta \delta_{\circ}}\right) \int_{\epsilon_{3}}^{\mathrm{R}_{3}} \mathrm{e}^{-\left(\frac{\delta_{0}-\delta}{\delta \delta_{\circ}}\right) t} \gamma(\rho, y, \mathrm{t}) d t\right\},
\end{aligned}
$$

now let $\epsilon_{3} \rightarrow 0$. Both terms on the right which depend on

$\epsilon_{3}$ approach a limit and

$\delta \int_{0}^{\mathrm{R} 3} \mathrm{k}(\rho, \mathrm{y}, \mathrm{t}) \mathrm{e}^{-\frac{\mathrm{t}}{\delta}} \mathrm{dt}=\frac{\delta}{\delta_{\circ}}\left\{\mathrm{e}^{-\left(\frac{\delta_{0}-\delta}{\delta \delta_{0}}\right) R_{3}} \gamma\left(\rho, y, \mathrm{R}_{3}\right)+\left(\frac{\delta_{\circ}-\delta}{\delta \delta_{\circ}}\right) \int_{0}^{\mathrm{R}_{3}} \mathrm{e}^{-\left(\frac{\delta_{0}-\delta}{\delta \delta_{\circ}}\right) \mathrm{t}} \gamma(\rho, y, \mathrm{t}) d t\right\}$

now let $R_{3} \rightarrow \infty$. If $s<s_{\circ}$, the first term on the right approaches zero.

$\delta \int_{0}^{\infty} \mathrm{k}(\rho, \mathrm{y}, \mathrm{t}) \mathrm{e}^{-\frac{t}{\delta}} \mathrm{dt}=\left(\frac{\delta_{\circ}-\delta}{\delta_{\circ}^{2}}\right) \int_{0}^{\infty} \mathrm{e}^{-\left(\frac{\delta_{0}-\delta}{\delta \delta_{\circ}}\right) t} \gamma(\rho, y, \mathrm{t}) d t, \quad$ for $\delta<\delta_{\circ}$,

the given theorem proved if the integral on the right converges.

Now by using the limit test for convergence (see [17]) we consider,

$$
\begin{aligned}
\lim _{t \rightarrow \infty} t^{2} \mathrm{e}^{-\left(\frac{\delta_{0}-\delta}{\delta \delta_{0}}\right) t} \gamma(x, y, t) & =\lim _{\mathrm{t} \rightarrow \infty} \frac{\mathrm{t}^{2}}{\mathrm{e}^{\left(\frac{\delta_{0}-\delta}{\delta \delta_{0}}\right) \mathrm{t}}}\left[\lim _{\mathrm{t} \rightarrow \infty} \gamma(x, y, t)\right] \\
& =0 *\left[\lim _{\mathrm{t} \rightarrow \infty} \gamma(x, y, t)\right]=0=\text { finite, }
\end{aligned}
$$

therefore, integral on the right of (12) converges for, $\delta<\delta_{\circ}$, hence, the given integral $\delta \int_{0}^{\infty} k(\rho, y, t) e^{-\frac{t}{\delta}} d t$ converges for $\delta<\delta_{\text {。 }}$.

The proof of the Theorem 2.1 is as follows:

$$
\begin{aligned}
\rho s \delta & \int_{0}^{\infty} \int_{0}^{\infty} \int_{0}^{\infty} \mathrm{e}^{-\frac{x}{\rho}-\frac{y}{s}-\frac{t}{\delta}} \emptyset(x, y, t) d x d y d t \\
& =\delta \int_{0}^{\infty} \mathrm{e}^{-\frac{\mathrm{t}}{\delta}}\left\{\rho \int_{0}^{\infty} \mathrm{e}^{-\frac{x}{\rho}}\left\{s \int_{0}^{\infty} \mathrm{e}^{-\frac{y}{s}} \emptyset(x, y, t) \mathrm{dy}\right\} d x\right\} d t \\
& =\delta \int_{0}^{\infty} \mathrm{e}^{-\frac{\mathrm{t}}{\delta}}\left\{\rho \int_{0}^{\infty} \mathrm{e}^{-\frac{x}{\rho}} h(x, s, t) d x\right\} d t \\
& =\delta \int_{0}^{\infty} \mathrm{e}^{-\frac{t}{\delta}} \mathrm{k}(\rho, \mathrm{y}, \mathrm{t}) \mathrm{dt},
\end{aligned}
$$

where,

$h(x, s, t)=\mathrm{s} \int_{0}^{\infty} \mathrm{e}^{-\frac{y}{s}} \emptyset(x, y, t) \mathrm{dy}$,

$\mathrm{k}(\rho, \mathrm{y}, \mathrm{t})=\rho \int_{0}^{\infty} \mathrm{e}^{-\frac{x}{\rho}} h(x, s, t) d x$

by Lemma 2.2, integral s $\int_{0}^{\infty} e^{\frac{y}{s}} \emptyset(x, y, t) d y$ converges for, $\mathrm{s}<\mathrm{s}_{0}$, also by Lemma 2.3 , integral $\rho \int_{0}^{\infty} \mathrm{e}^{-\frac{\mathrm{x}}{\rho}} \mathrm{h}(\mathrm{x}, \mathrm{s}, \mathrm{t}) \mathrm{dx}$ converges for $\rho<\rho_{\circ}$, and by Lemma 2.4, integral $\delta \int_{0}^{\infty} e^{-\frac{t}{\delta}} k(\rho, y, t) d t$ converges for $\delta<\delta_{\circ}$, therefore, integral in the RHS (15) converges for $\rho<\rho_{\circ}, s<s_{\circ}$ and $\delta<\delta_{\circ}$, hence the integral: $\rho s \delta \int_{0}^{\infty} \int_{0}^{\infty} \int_{0}^{\infty} \mathrm{e}^{-\frac{x}{\rho}-\frac{y}{s}-\frac{t}{\delta}} \emptyset(x, y, t) d x d y d t$, converges for, $\rho<\rho_{\circ}, s<s_{\circ}$ and $\delta<\delta_{o \text { " }}$ then the proof of the Theorem 2.1 is completed.

Corollary 2.4 If the integral (1) diverges at $\rho=\rho_{\circ}$, $\mathrm{s}=\mathrm{s}_{\mathrm{o}}$ and $\delta=\delta_{\circ}$, then the integral, (1) diverges at

$\rho<\rho_{\circ}, s<s_{\circ} \delta<\delta_{\circ}$,

Corollary 2.5 The region of the convergence of the integral (1) is the positive quadrant of the xyt - plane. Now we prove absolute convergence of integral (1).

Theorem 2.6 If the integral (1) converges absolutely at $\rho=\rho_{\circ}, s=s_{0}$ and $\delta=\delta_{\circ}$, then integral (1) converges absolutely for $\rho<\rho_{\circ}$, $s<s_{\circ}$ and $\delta<\delta_{\circ}$,

Proof We know that: 
$\mathrm{e}^{-\frac{\mathrm{x}}{\rho_{0}}-\frac{\mathrm{y}}{s_{0}}-\frac{\mathrm{t}}{\delta_{0}}}|\emptyset(x, y, t)| \leq \mathrm{e}^{-\frac{\mathrm{x}}{\rho}-\frac{\mathrm{y}}{\mathrm{s}}-\frac{\mathrm{t}}{\delta}}$

for $\left(\rho \leq \rho_{\circ}<\infty, s \leq s_{\circ}<\infty, \delta \leq \delta_{\circ}<\infty\right)$,

therefore,

$$
\begin{array}{r}
\rho_{\circ} s_{\circ} \delta_{\circ} \int_{0}^{\infty} \int_{0}^{\infty} \int_{0}^{\infty} \mathrm{e}^{-\frac{x}{\rho_{\mathrm{o}}}-\frac{y}{s_{\mathrm{o}}}-\frac{\mathrm{t}}{\delta_{\mathrm{o}}}}|\emptyset(x, y, t)| d x d y d t \\
\leq \rho s \delta \int_{0}^{\infty} \int_{0}^{\infty} \int_{0}^{\infty} \mathrm{e}^{-\frac{x}{\rho}-\frac{y}{\mathrm{~s}}-\frac{\mathrm{t}}{\delta}}|\emptyset(x, y, t)| d x d y d t
\end{array}
$$

form given hypothesis, $\rho s \delta \int_{0}^{\infty} \int_{0}^{\infty} \int_{0}^{\infty} \mathrm{e}^{-\frac{x}{\rho}-\frac{y}{s}-\frac{t}{\delta}}|\emptyset(x, y, t)| d x d y d t$, converge. Hence, we have: $\rho_{\circ} s_{\circ} \delta_{0} \int_{0}^{\infty} \int_{0}^{\infty} \int_{0}^{\infty} e^{-\frac{x}{\rho_{0}}-\frac{y}{s_{0}}-\frac{t}{\delta_{0}}}$ $|\emptyset(x, y, t)| d x d y d t$ converge for $\left(\rho \leq \rho_{\circ}, \mathrm{s} \leq \mathrm{s}_{\circ}, \delta \leq \delta_{\circ}\right)$.

Therefore the integral (1) converges absolutely for, $\left(\rho \leq \rho_{\circ}, s \leq s_{\circ}, \delta \leq \delta_{\circ}\right)$.
Lemma 3.2 If $\mathrm{g}(\mathrm{x}, \mathrm{y}, \mathrm{t})=\mathrm{s}_{\circ} \int_{0}^{\mathrm{y}} \mathrm{e}^{-\frac{\mathrm{v}}{s_{\circ}}} f(x, v, t) \mathrm{dv}$ is bounded on $[0, \infty)$ then the Elzaki transform of, $f$ with respect to s converges uniformly on $[\mathrm{s}, \infty)$ if $\mathrm{s}<\mathrm{s}$.

Proof If $0 \leq r \leq r_{1}$ then consider,

$$
\begin{aligned}
& s \int_{r}^{r_{1}} f(x, y, t) e^{-\frac{y}{s}} d y=s \int_{r}^{r_{1}} e^{-\left(\frac{s_{0}-s}{s s_{o}}\right) y} f(x, y, t) e^{\frac{y}{s_{0}}} d y \\
& =\frac{s}{\mathrm{~s}_{0}} \int_{\mathrm{r}}^{\mathrm{r}_{1}} \mathrm{e}^{-\left(\frac{\mathrm{s}_{0}-\mathrm{s}}{\mathrm{s}_{\mathrm{o}}}\right) \mathrm{y}} g_{y}(x, y, t) d y,
\end{aligned}
$$

using integration by parts,

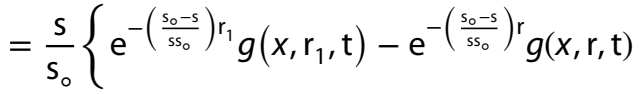

$$
\begin{aligned}
& \left.+\left(\frac{\mathrm{s}_{\circ}-\mathrm{s}}{\mathrm{ss}_{\circ}}\right) \int_{\mathrm{r}}^{\mathrm{r}_{1}} \mathrm{e}^{-\left(\frac{\mathrm{s}_{\mathrm{o}}-\mathrm{s}}{\mathrm{s} \mathrm{s}_{\mathrm{o}}}\right) \mathrm{y}} g(x, y, t) d y\right\},
\end{aligned}
$$

$$
\begin{aligned}
\left|s \int_{r}^{r_{1}} e^{-\frac{y}{s}} f(x, y, t) d y\right| & \leq M\left\{e^{-\left(\frac{s_{0}-s}{s s_{0}}\right) r_{1}}+e^{-\left(\frac{s_{0}-s}{s s_{0}}\right) r}+\left(\frac{s_{\circ}-s}{s s_{\circ}}\right) \int_{r}^{r_{1}} e^{-\left(\frac{s_{0}-s}{s s_{0}}\right) y} d y\right\} \\
& =M\left\{e^{-\left(\frac{s_{0}-s}{s s_{0}}\right) r_{1}}+e^{-\left(\frac{s_{0}-s}{s s_{0}}\right) r}-e^{-\left(\frac{s_{0}-s}{s s_{0}}\right) r_{1}}+e^{-\left(\frac{s_{0}-s}{s s_{\circ}}\right) r}\right\} \\
& =2 M e^{-\left(\frac{s_{0}-s}{s s_{0}}\right) r} \text { for } s \leq s_{\circ},
\end{aligned}
$$

\section{Uniform convergence}

In this section, we prove the uniform convergence of triple Elzaki Transform.

Theorem 3.1 If $\mathrm{f}(\mathrm{x}, \mathrm{y}, \mathrm{t})$ is continuous on $[0, \infty) \mathrm{x}$ $[0, \infty) \times[0, \infty)$, and,

$H(x, y, t)=\rho_{\circ} s_{\circ} \delta_{\circ} \int_{0}^{x} \int_{0}^{y} \int_{0}^{t} \mathrm{e}^{-\frac{u}{\rho_{\circ}}-\frac{v}{s_{\circ}}-\frac{w}{\delta_{\circ}}} f(u, v, w) d u d v d w$

is bounded on $[0, \infty) \times[0, \infty) \times[0, \infty)$ then the triple of Elzaki transform of, $f$ converges uniformly on $[\rho, \infty) \times[s, \infty) \times[\delta, \infty)$ if, $\rho<\rho_{\circ}, s<s_{\circ}, \delta<\delta_{\circ}$. For the proof, we will use the following lemmas: by Cauchy criterion for uniform convergence [18], $\mathrm{s} \int_{\mathrm{r}}^{\mathrm{r}_{1}} f(x, y, t) \mathrm{e}^{-\frac{\mathrm{y}}{\mathrm{s}}} \mathrm{dy}$, converges uniformly on $[\mathrm{s}, \infty)$ if $\mathrm{s}<\mathrm{s}_{\mathrm{o}}$.

Hence, Elzaki transform of, $f$ with respect to, $s$ converges uniformly on $[s, \infty)$ if $s<s_{0}$.

Lemma 3.3 If $g(x, y, t)=\rho_{\circ} s_{\circ} \int_{0}^{x} \int_{0}^{y} e^{-\frac{u}{\rho_{o}}-\frac{v}{s_{\circ}}} f(u, v, t) d u d v$ is bounded on $[0, \infty) \times[0, \infty)$ then the Elzaki transform of, $f$ with respect to $\rho, s$ converges uniformly on $[\rho, \infty) \times[s, \infty)$ if $\mathrm{s}<\mathrm{s}$ oproof see double Elzaki transform [7].

Lemma 3.4 If the integral $g(\rho, \mathrm{s}, \mathrm{t})=\rho \mathrm{s} \int_{0}^{\infty} \int_{0}^{\infty}$ $\mathrm{e}^{-\frac{\mathrm{x}}{\rho}-\frac{\mathrm{y}}{\mathrm{s}}} f(x, y, t) d x d y, \quad \rho<\rho_{\circ}, \mathrm{s}<\mathrm{s}$ 。 and,$a(\rho, \mathrm{s}, \mathrm{t})=\delta_{\circ} \int_{0}^{\mathrm{t}}$ $\mathrm{e}^{-\frac{\mathrm{w}}{\delta_{\circ}}} \mathrm{g}(\rho, \mathrm{s}, \mathrm{w}) \mathrm{dw}$ is bounded on $[0, \infty)$, then Elzaki transform of the function $f$ with respect to $\rho$, s converges uniformly on $[\rho, \infty) \times[s, \infty)$. 
Proof The proof of Theorem 3.1 is as follows,

$\mathrm{H}(\mathrm{x}, \mathrm{y}, \mathrm{t})=\rho_{\circ} \mathrm{s}_{\circ} \delta_{\circ} \int_{0}^{x} \int_{0}^{y} \int_{0}^{t} \mathrm{e}^{-\frac{u}{\rho_{\circ}}-\frac{v}{s_{\circ}}-\frac{w}{\delta_{\circ}}} f(u, v, w) d u d v d w$

$$
=\delta_{\circ} \int_{0}^{t} \mathrm{e}^{-\frac{w}{\delta_{\circ}}}\left\{\rho_{\circ} \mathrm{s}_{\circ} \int_{0}^{x} \int_{0}^{y} \mathrm{e}^{-\frac{u}{\rho_{\circ}}-\frac{v}{s_{\circ}}} f(u, v, w) d u d v\right\} d w=\delta_{\circ} \int_{0}^{t} \mathrm{e}^{-\frac{w}{\delta_{\circ}}} g(x, y, w) d w,
$$

where $\quad g(\mathrm{x}, \mathrm{y}, \mathrm{w})=\rho_{\circ} \mathrm{s}_{\circ} \int_{0}^{x} \int_{0}^{y} \mathrm{e}^{-\frac{\mathrm{u}}{\rho_{\circ}}-\frac{\mathrm{v}}{\mathrm{s}_{\circ}}} f(u, v, w) d u d v$ is bounded on $[0, \infty) \times[0, \infty)$, by Lemmas 3.2, and 3.3 double Elzaki transform of $f$ with respect to $\rho, s$ converges uniformly on $[\rho, \infty) \times[s, \infty)$ if $\rho<\rho_{\circ}, s<s_{\circ}$.

Also by Lemma 3.4, Elzaki transform of $g$ with respect to $\delta$ converges uniformly on $[\delta, \infty)$ if $\delta<\delta_{\circ}$.

Hence triple Elzaki transform of, $f$ converges uniformly on $[\rho, \infty) \times[s, \infty) \times[\delta, \infty)$ if $\rho<\rho_{\circ}, s<s_{\circ}$ and $\delta<\delta_{\circ}$.

We now prove the differentiability of triple Elzaki transform.

Theorem 3.5 If $f(x, y, t)$ is continuous on $[0, \infty) \times$ $[0, \infty) \times[0, \infty)$ and,

$\mathrm{H}(\mathrm{x}, \mathrm{y}, \mathrm{t})=\rho_{\circ} \mathrm{s}_{\circ} \delta_{\circ} \int_{0}^{x} \int_{0}^{y} \int_{0}^{t} e^{-\frac{u}{\rho_{\circ}}-\frac{v}{s_{\circ}}-\frac{w}{\delta_{\circ}}} f(u, v, w) d u d v d w$,

is bounded on $[0, \infty) \times[0, \infty) \times[0, \infty)$, then the triple Elzaki transform of the fuction $f$ is infinitely differentiable with respect to $\rho$, s and $\delta$ on $[\rho, \infty) \times[s, \infty) \times[\delta, \infty) \rho<\rho_{\circ}$, $s<s_{o}$, and $\delta<\delta_{\circ}$ with $\frac{\partial^{m+n}}{\partial \rho^{m} \partial s^{n}} \bar{f}(\rho, s, \delta)=(-1)^{\mathrm{m}+\mathrm{n}+\mathrm{k}} \rho s \delta \int_{0}^{\infty} \int_{0}^{\infty} \int_{0}^{\infty}$

$\mathrm{e}^{-\frac{\mathrm{x}}{\rho}-\frac{\mathrm{y}}{\mathrm{s}}-\frac{\mathrm{t}}{\delta}} x^{m} y^{n} t^{k} f(x, y, t) d x d y d t$

for the proof, we will use the following lemmas.

Lemma 3.6 If $\mathrm{g}(\mathrm{x}, \mathrm{y}, \mathrm{t})=\int_{0}^{y} \mathrm{e}^{-\frac{\mathrm{v}}{s_{\mathrm{o}}}} f(x, v, t) \mathrm{dv}$ is bounded on $[0, \infty)$ then the Elzaki transform of is infinitely differentiable with respect to $s$ on $[\mathrm{s}, \infty)$ if $\mathrm{s}<\mathrm{s}$, with,

$\frac{\partial^{n}}{\partial s^{n}} \bar{f}(\mathrm{x}, \mathrm{s})=(-1)^{\mathrm{n}} \int_{0}^{\infty} \mathrm{e}^{-\frac{y}{s}} y^{n} f(x, y, t) d y$

Proof Foremost we will prove that the integrals,

$I_{n}(x, s, t)=(-1)^{n} \int_{0}^{\infty} e^{-\frac{y}{s}} y^{n} f(x, y, t) d y, \quad n=0,1,2,3, \ldots$,

all converge uniformly on $[s, \infty)$ if $s<s_{\circ}$ and if $0 \leq r \leq r_{1}$, then,

$$
\begin{aligned}
s \int_{r}^{r_{1}} e^{-\frac{y}{s}} y^{n} f(x, y, t) d y & =s \int_{r}^{r_{1}} e^{-\left(\frac{s_{0}-s}{s s_{0}}\right) y} y^{n} g_{y}(x, y, t) d y \\
& =\frac{s}{s_{0}}\left\{e^{-\left(\frac{s_{0}-s}{s s_{0}}\right) r_{1}} r_{1}^{n} g\left(x, r_{1}, t\right)-e^{-\left(\frac{s_{0}-s}{s s_{0}}\right) r} r^{n} g(x, r, t)-\int_{r}^{r_{1}}\left[\frac{d}{d t} e^{-\left(\frac{s_{0}-s}{s s_{0}}\right) y} y^{n}\right] g(x, y, t) d y\right\},
\end{aligned}
$$

therefore, if $|(y),| \leq<\infty$ on $[0, \infty)$ then,

$$
\begin{aligned}
& \left|s \int_{r}^{r_{1}} e^{-\frac{y}{s}} y^{n} f(x, y, t) d y\right| \leq M\left\{e^{-\left(\frac{s_{0}-s}{s s_{0}}\right) r_{1}} r_{1}^{n}+e^{-\left(\frac{s_{0}-s}{s s_{0}}\right) r} r^{n}-e^{-\left(\frac{s_{0}-s}{s s_{0}}\right) r_{1}} r_{1}^{n}+e^{-\left(\frac{s_{0}-s}{s s_{0}}\right) r} r^{n}\right\} \\
& \left|s \int_{r}^{r_{1}} e^{-\frac{y}{s}} y^{n} f(x, y, t) d y\right| \leq 2 M e^{-\left(\frac{s_{0}-s}{s s_{0}}\right) r} r^{n} \text { for } 0 \leq r \leq r_{1} .
\end{aligned}
$$


By Cauchy criterion for uniform convergence $[18]]_{n}(x, s, t)$ converges uniformly on $[s, \infty)$ if $s<s$. Now, using Trench [18] and induction proof, we have (17). That is Elzaki transform of $f$ is infinitely differentiable with respect to $s$, on $[s, \infty)$ if $s<s_{0}$.

Lemma 3.7 If $g(x, y, t)=\rho_{\circ} s_{\circ} \int_{0}^{x} \int_{0}^{y} e^{-\frac{u}{\rho_{o}}-\frac{v}{s_{o}}} f(u, v, w) d u d v$ is bounded on $[0, \infty) \times[0, \infty)$ then double Elzaki transform of $f$ is infinitely differentiable with respect to $\rho, s$ on $[\rho, \infty) \times[s, \infty)$ if $\rho<\rho_{\circ}, s<s_{\circ}$ with,

$\frac{\partial^{m+n}}{\partial \rho^{m} \partial s^{n}} \bar{f}(\rho, s, t)=(-1)^{m+n} \rho s \int_{0}^{\infty} \int_{0}^{\infty} e^{-\frac{x}{\rho}-\frac{y}{s}} x^{m} y^{n} f(x, y, t) d x d y$,

for the proof see double Elzaki transform [7].

Lemma 3.8 If the integral $\emptyset(\rho, s, t)=\rho s \int_{0}^{\infty} \int_{0}^{\infty} e^{-\frac{x}{\rho}-\frac{y}{s}}$ $x^{m} y^{n} f(x, y, t) d x d y$ converges uniformly on $[\rho, \infty),[s, \infty)$ if $\rho<\rho_{\circ}, \mathrm{s}<\mathrm{s}_{\circ}$ and $\mathrm{h}(\rho, \mathrm{s}, \mathrm{t})=\delta \int_{0}^{\mathrm{t}} \mathrm{e}^{-\frac{\mathrm{w}}{\delta_{\circ}}} \emptyset(\rho, \mathrm{s}, \mathrm{t}) \mathrm{dt}$ is bounded on $[0, \infty)$ then the Elzaki transform of $\emptyset$ is infinitely differentiable with respect to $\delta$ on $[\delta, \infty)$ if $\delta<\delta_{\alpha}$ with the,

$\frac{\partial^{k}}{\partial \delta^{k}} \emptyset(\rho, \mathrm{s}, \mathrm{t})=(-1)^{\mathrm{k}} \int_{0}^{\infty} \mathrm{e}^{-\frac{\mathrm{t}}{\delta}} t^{k} \emptyset(\rho, \mathrm{s}, \mathrm{t}) d t$

Proof The validation is a similar to Lemmas 3.6 and 3.7.

The validation of the Theorem 3.5 is as follows:

$H(x, y, t)=\rho_{\circ} s_{\circ} \delta_{\circ} \int_{0}^{x} \int_{0}^{y} \int_{0}^{t} e^{-\frac{u}{\rho_{0}}-\frac{v}{s_{0}}-\frac{w}{\delta_{0}}} f(u, v, w) d u d v d w$

$$
=\delta_{\circ} \int_{0}^{t} e^{-\frac{w}{\delta_{\circ}}} g(x, y, w) d w
$$

where $g(x, y, w)=\rho_{0} s_{0} \int_{0}^{x} \int_{0}^{y} e^{-\frac{u}{\rho_{o}}-\frac{v}{s_{\circ}}} f(u, v, w) d u d v$, is bounded on $[0, \infty) \times[0, \infty)$. By Lemma 3.7, Elzaki transform of $f$ is infinitely differentiable with respect to $\rho, s$ on $[\rho, \infty),[s, \infty)$ if $\rho<\rho_{\circ}, s<s_{\circ}$, also by Lemma 3.8, Elzaki transform of $g$ is infinitely differentiable with respect to $\delta$ on $[\delta, \infty)$ if $\delta<\delta_{\text {o }}$.

Hence triple Elzaki transform of $f$ is infinitely differentiable with respect to $\rho, s$ and $\delta$ on $[\rho, \infty) \times[s, \infty) \times$ $[\delta, \infty) \rho<\rho_{\circ}, s<s_{\circ}$, and $\delta<\delta_{\circ}$.

\section{Triple Elzaki transform of triple integral}

Now we find the triple Elzaki transform of triple integral.

Theorem 4.1 If $\mathrm{E}_{\mathrm{x}} \mathrm{E}_{\mathrm{y}} \mathrm{E}_{\mathrm{t}} f(x, y, t)=\bar{f}(p, s, \delta)$ and,

$g(\mathrm{x}, \mathrm{y}, \mathrm{t})=\int_{0}^{\mathrm{x}} \int_{0}^{\mathrm{y}} \int_{0}^{\mathrm{t}} f(u, v, w) d u d v d w$,

then: $\mathrm{E}_{\mathrm{x}} \mathrm{E}_{\mathrm{y}} \mathrm{E}_{\mathrm{t}}\left\{\int_{0}^{\mathrm{x}} \int_{0}^{\mathrm{y}} \int_{0}^{\mathrm{t}} f(u, v, w) d u d v d w\right\}=\rho s \delta \overline{\mathrm{f}}(\mathrm{p}, \mathrm{s}, \delta)$.

Proof If we denote, $\mathrm{h}(\mathrm{x}, \mathrm{y}, \mathrm{t})=\int_{0}^{\mathrm{y}} f(\mathrm{x}, \mathrm{v}, \mathrm{t}) \mathrm{dv}$, by using fundamental theorem of calculous,

$h_{y}(x, y, t)=f(x, y, t)$,

since,

$h(x, 0, t)=0$,

taking double Elzaki transform of Eq. (22), we get:

$\overline{\mathrm{h}}(\rho, s, \delta)=s \bar{f}(\rho, s, \delta)$,

from Eq. (20) we get,

$r(\mathrm{x}, \mathrm{y}, \mathrm{t})=\int_{0}^{\mathrm{t}} \mathrm{h}(\mathrm{x}, \mathrm{y}, \mathrm{w}) \mathrm{dw}$,

$r_{t}(x . y, t)=\mathrm{h}(\mathrm{x} . \mathrm{y}, \mathrm{t})$ and $\mathrm{r}(\mathrm{x}, \mathrm{y}, 0)=0$,

$\bar{r}(\rho, s, \delta)=\delta \bar{h}(\rho, s, \delta)$,

$g(x, y, t)=\int_{0}^{x} r(u, y, t) d u$, and $g(0, y, t)=0$,

$\bar{g}(\rho, s, \delta)=\rho \bar{r}(\rho, s, \delta)$,

now by using (24), (25) and (26), we obtain,

$\mathrm{E}_{\mathrm{x}} \mathrm{E}_{\mathrm{y}} \mathrm{E}_{\mathrm{t}}\left\{\int_{0}^{\mathrm{x}} \int_{0}^{\mathrm{y}} \int_{0}^{\mathrm{t}} f(u, v, w) d u d v d w\right\}=\rho s \delta \bar{f}(\mathrm{p}, \mathrm{s}, \delta)$. 


\section{Application of triple Elzaki transform in Volterra integro-partial differential equation}

In this section, we use the triple Elzaki transform to solve the Volterra integro-partial differential equation, which already solved in [19] by means of differential transform method.

Example 5.1 Consider the Volterra integro-partial differential equation,

$$
\begin{aligned}
& \frac{\partial u(x, y, t)}{\partial x}+\frac{\partial u(x, y, t)}{\partial y}+\frac{\partial u(x, y, t)}{\partial t}=-x^{2} y^{2} t^{2}+4 x y+4 x t \\
& +4 y t+2 \int_{0}^{x} \int_{0}^{y} \int_{0}^{t} u(k, r, s) d k d r d s
\end{aligned}
$$

with,

$\mathrm{u}(\mathrm{x}, \mathrm{y}, 0)=0, \mathrm{u}(\mathrm{x}, 0, t)=0, \mathrm{u}(0, y, t)=0$,

applying triple Elzaki transform of Eq. (27) to get,

$$
\begin{aligned}
& \frac{1}{\sigma} \overline{\mathrm{u}}(\rho, s, \delta)-\sigma \overline{\mathrm{u}}(0, s, \delta)+\frac{1}{\rho} \overline{\mathrm{u}}(\rho, s, \delta)-\rho \overline{\mathrm{u}}(\rho, 0, \delta) \\
& \quad+\frac{1}{\delta} \overline{\mathrm{u}}(\rho, s, \delta)-\delta \overline{\mathrm{u}}(\rho, s, 0)=-8 \rho^{4} s^{4} \delta^{4}+4 \rho^{3} s^{3} \delta^{2} \\
& \quad+4 \rho^{3} s^{2} \delta^{3}+4 \rho^{2} s^{3} \delta^{3}+\rho s \delta \overline{\mathrm{u}}(\rho, s, \delta),
\end{aligned}
$$

the double Elzaki transforms of Eq. (28)

$\overline{\mathrm{u}}(0, s, \delta)=0, \overline{\mathrm{u}}(\rho, 0, \delta)=0, \overline{\mathrm{u}}(\rho, s, 0)=0$,

substituting (30) in (29), simplifying, and we obtain,

$$
\begin{aligned}
\frac{1}{\sigma} \overline{\mathrm{u}}(\rho, s, \delta)+\frac{1}{\rho} \overline{\mathrm{u}}(\rho, s, \delta)+\frac{1}{\delta} \overline{\mathrm{u}}(\rho, s, \delta) \\
=-8 \rho^{4} s^{4} \delta^{4}+4 \rho^{3} s^{3} \delta^{2} \\
\quad+4 \rho^{3} s^{2} \delta^{3}+4 \rho^{2} s^{3} \delta^{3}+\rho s \delta \overline{\mathrm{u}}(\rho, s, \delta)
\end{aligned}
$$

So $\bar{u}(\rho, s, \delta)=4 \rho^{3} s^{3} \delta^{3}$, applying triple inverse Elzaki transform, to ascertain the exact solution in the shape,

$u(x, y, t)=4 x y t$.

Which is the same result that obtained by using differential transform method, see [19].

To compere this method with other methods you can refer to papers $[1,19]$, because the main objectives of this paper is to study the convergence, absolute convergence and uniform convergence of triple Elzaki transform.

\section{Conclusion}

We have demonstrated the convergence, absolute convergence and uniform convergence of triple Elzaki transform they have been shown. Other than these, we got triple Elzaki transform of triple integral and use to illuminate Volterra integro-partial differential equation.

\section{Compliance with ethical standards}

Conflict of interest The authors declare that they have no conflict of interest.

Open Access This article is distributed under the terms of the Creative Commons Attribution 4.0 International License (http://creativeco mmons.org/licenses/by/4.0/), which permits unrestricted use, distribution, and reproduction in any medium, provided you give appropriate credit to the original author(s) and the source, provide a link to the Creative Commons license, and indicate if changes were made.

\section{References}

1. Di Atangana A (2013) A note on the triple laplace transform and its applications to some kind of third-order differential equation. Abstr Appl Anal 2013:10

2. Chamekh M, Elzaki TM, Brik N (2019) Semi-analytical solution for some proportional delay differential equations. SN Appl Sci 1:148. https://doi.org/10.1007/s42452-018-0130-8

3. Elzaki TM, Ezaki SM (2011) Applications of new transform 'Elzaki Transform' to partial differential equations. Glob J Pure Appl Math 7:65-70

4. Elzaki TM, Ezaki SM (2011) On the Elzaki transform and ordinary differential equation with variable coefficients. Adv Theor Appl Math 6:41-46

5. Elzaki TM, Biazar J (2013) Homotopy perturbation method and Elzaki transform for solving systems of nonlinear partial differential equations. World Appl Sci J 24:944-948

6. Elzaki TM, Ezaki SM, Hilal EMA (2012) ELzaki and Sumudu transform for solving some differential equations. Glob J Pure Appl Math 8:167-173

7. Idrees MI, Ahmed Z (2018) On the convergence of double Elzaki transform. Int J Adv Appl Sci 5(6):19-24

8. Alderremy AA, Elzaki TM, Chamekh M (2018) New transform iterative method for solving some Klein-Gordon equations. Results Phys 10:655-659. https://doi.org/10.1016/j.rinp.2018.07.004

9. Ziane D, Elzaki TM, Cherif MH (2018) Elzaki transform combined with variational iteration method for partial differential equations of fractional order. Fundam J Math Appl 1(1):102-108

10. Elzaki TM, Alderremy AA (2018) On the new double integral transform for solving singular system of hyperbolic equations. J Nonlinear Sci Appl 11:1207-1214. www.isr-publications.com/ jnsa

11. Jena RM, Chakraverty S (2018) Solving time-fractional NavierStokes equations using homotopy perturbation Elzaki 
transform. SN Appl Sci 1:16. https://doi.org/10.1007/s4245 2-018-0016-9

12. Eltayeb $H$, Kilicman A (2010) On some applications of a new integral transform. Int J Math Anal 4:123-132

13. Belgacem FBM (2006) Introducing and analyzing deeper Sumudu properties. Nonlinear Stud 13(1):23-41

14. Watugala GK (1993) Sumudu transform: a new integral transform to solve differential equations and control engineering problems. Integr Educ 24(1):35-43

15. Belgicus FBM (2010) Sumudu transform applications to Bessel functions and equations. Appl Math Sci 4(74):3665-3686

16. Belgacem FBM, Al-Shemas EHN (2014) Towards a Sumudu based estimation of large-scale disasters environmental fitness changes adversely affecting population dispersal and persistence. In: The AIP conference proceedings, AIP, vol 1637, no 1, pp 1442-1449. https://doi.org/10.1063/1.4907311

17. Widder DV (2005) Advanced Calculus, 2nd edn. Prentice Hall of India Private Limited, New Delhi
18. Trench WF (2012) Functions defined by improper integrals. https ://faculty.ksu.edu.sa/fawaz/File282/Books/TRENCH_IMPROPER_ FUNCTIONS.pdf

19. Moghadam MM, Saeedi H (2010) Application of differential transforms for solving the Volterra integro-partial differential equations. Iranian Journal of Science and Technology, Transaction A 34(A1):59

20. Belgacem FBM (2007) Applications of Sumudu transform to indefinite periodic parabolic equations. In: 6th international conference on mathematical problems and aerospace sciences, (ICNPAA 06). Cambridge Scientific, Watertown

Publisher's Note Springer Nature remains neutral with regard to jurisdictional claims in published maps and institutional affiliations. 